\title{
Los sistemas urbanos de cuenca en México. Transitando a estrategias integrales de gestión hídrica
}

\section{Urban systems of river basin in Mexico. Setting up integral strategies of water resources management}

Rafael Monroy-Ortiz*

\begin{abstract}
Environment degradation has gradually become a systemic problem, which affects all aspects of life. Consequently, definition of public policies must take this factor into account. The aim of this paper is studying possible strategies for mitigation and an adequate urban system in Mexico for water availability that appears to be reduced. These policies mean to set up local integrated water resources management. In order to do that, it is proposed the territorial unit called urban system of river basin. Prevailing regional conditions demonstrates disparity in two senses: there is a high economical activity concentration, and an increased environmental vulnerability caused by territorial fragmentation and over extraction of water resources. Both factors must be integrated into public policies because of their relevance for the country structural economy, and the prevailing society conditions.
\end{abstract}

Keywords: integrated water resources management, urban systems of river basin, economical vulnerability, territorial particularities, territorial policies.

\section{Resumen}

El deterioro del sistema natural se ha vuelto un problema sistémico que afecta todas las esferas de la vida, y en consecuencia es un factor que condiciona la definición de las políticas públicas. En este trabajo se abordan las posibles estrategias de mitigación y adecuación para el sistema urbano en México, dadas las condiciones de emergencia del agua. Éstas tienen que ver con el manejo integrado de recursos hídricos, mismo que se analiza en términos del sistema urbano de cuenca. Las condiciones prevalecientes demuestran una disparidad regional dada la alta concentración de la actividad económica, pero también una creciente vulnerabilidad ambiental ocasionada por la fragmentación territorial y la sobreextracción del recurso. Ambos factores deben incorporarse a la política pública debido a su relevancia para la estructura económica del país, así como por las condiciones prevalecientes en la sociedad.

Palabras clave: manejo integrado de recursos hídricos, sistema urbano de cuenca, vulnerabilidad económica, particularidades territoriales, políticas territoriales.

* Universidad Autónoma del Estado de Morelos, México. Correo-e: rafaelmoor@hotmail.com, moorafael@yahoo.com.mx. 


\section{Introducción}

La actividad económica genera múltiples efectos en el ambiente; los patrones de producción y consumo llevan al aprovechamiento intensivo de recursos naturales y energéticos (Martínez y Roca, 2001: 14). En consecuencia, se emiten gases de efecto invernadero y se modifica la cubierta terrestre, lo que contribuye en el cambio climático (IPCC, 2007b: 5).

En términos económicos, la pérdida de recursos y la contaminación son efectos que se internalizan, o en su caso se resuelven con bienes sustitutivos, desarrollos tecnológicos y con la exploración de nuevas fuentes. Sin embargo, los efectos ambientales negativos implican el incremento de los costos de producción (O'Connor, 2001: 218; Altvater y Manhkopf, 2002: 307), así como la vulnerabilidad social.

Dicha vulnerabilidad se caracteriza por la dificultad para proveerse alimento, agua o condiciones de vida saludables (UNEP, 2007: 7); pero también por la incapacidad para resarcir los efectos de los eventos naturales (CEPAL, 2009: 28). Por su naturaleza social, los efectos más relevantes del cambio climático son la disminución del rendimiento agrícola, la agudización del estrés hídrico y los problemas de salud humana.

En términos de alimentación, las personas en condiciones de hambre y subnutrición alcanzaron los 1,000 millones en 2009 (FAO, 2011: 71), es decir, una de cada siete personas en el planeta. Por otro lado, la misma proporción de personas carece de agua suficiente para mantener su salud (IFPRI-IWMI, 2002: 2). Incluso, se estima que una décima parte de las enfermedades registradas en el planeta las ocasionan problemas en la calidad y cantidad de agua (UNESCO, 2009: 140).

En particular, el sistema urbano contribuye de manera relevante con el deterioro ambiental global. De hecho, la intensidad de la actividad económica y las necesidades sociales de las aglomeraciones humanas son los principales responsables de la emisión de gases de efecto invernadero, así como de la modificación de la cubierta terrestre. En este sentido, las ciudades generan entre 50 y $80 \%$ del producto interno bruto (РІв) en los países más urbanizados (OCDE, 2006: 305; un-Habitat, 2006: 17), y albergan a la mitad de la población mundial (UNPF, 2009: 70).

La consolidación de la ciudad como espacio económico, así como la tasa de crecimiento urbano estimado para las siguientes décadas sugieren el incremento de la demanda de recursos, pero también de la vulnerabilidad social que en ella persiste. En este sentido, existe consenso para coordinar la atención a aspectos de alimentación, salud, educación o ambientales (PNUD, 2010). En términos urbanos, se considera necesario generar una agenda común que atienda el cambio climático, la crisis económica, el aprovechamiento de las diversas energías, la seguridad 
alimentaria, la desigualdad del ingreso, las dimensiones de las ciudades y la diversidad cultural (Un-Habitat, 2009: 75).

A pesar de ello, la agenda urbana en México cumple principalmente las funciones de flexibilizar la apropiación de reservas territoriales e intervenir en los patrones de ocupación del suelo (Azuela, 1989: 204); es decir, se compone de políticas de corte funcionalista caracterizadas por procurar el crecimiento urbano y organizar económicamente el espacio (Asuad et al., 2007: 358; Mendoza y Díaz, 2006: 33), aspectos que sólo sirven para promover la creciente vulnerabilidad social o ambiental.

$\mathrm{Al}$ respecto, el crecimiento nacional del PIB en México es de los menores respecto a la región más pobre del continente, y la cantidad de personas en situación de pobreza es equivalente al 31.7\% de la población total, mientras que $8.7 \%$ está en el rango de indigencia (CEPAL, 2008: 226); es decir, uno de cada tres habitantes urbanos muestra un signo de vulnerabilidad.

Por otro lado, $95 \%$ del territorio no cuenta con política urbana alguna (Monroy-Ortiz y Monroy, 2007: 283). Además, los instrumentos urbanos no se actualizan periódicamente; ejemplo de ello es que la Ley de Asentamientos Humanos se modificó 20 años después de su primera publicación (LGAH, 1994) o que solamente 5\% de los programas de desarrollo urbano de centro de población habían sido actualizados después del año 2000.

\section{Políticas urbanas no convencionales y el sistema urbano de cuenca (suc)}

Las políticas urbanas en México contemplan dos objetivos generales: la competitividad económica y la generación de reservas territoriales para la expansión urbana. Ambos se consideran útiles para proveer desarrollo urbano, de hecho son temas centrales en la Ley General de Asentamientos Humanos (1994) y en los programas de desarrollo urbano.

En particular, el Programa Nacional de Desarrollo Urbano y Ordenamiento del Territorio (2001) propone

Maximizar la eficiencia económica del territorio garantizando su cohesión social y cultural.

Integrar un Sistema Urbano Nacional en sinergia con el desarrollo regional en condiciones de sustentabilidad: gobernabilidad territorial, eficiencia y competitividad económica, cohesión social y cultural, y planificación y gestión urbana. Integrar el suelo urbano apto para desarrollo como instrumento de soporte para la expansión urbana por medio de satisfacer los requerimientos de suelo para la vivienda y el desarrollo urbano (Sedesol, 2001: 88). 
Dado el contexto de emergencia, queda pendiente asumir mayor responsabilidad en la atención a variables de corte ambiental y social. Es decir, es necesario mitigar los efectos del cambio climático, plantear esquemas alternos de aprovechamiento de energías o, en su defecto, atender problemas de vulnerabilidad social, como la seguridad alimentaria, la desigualdad del ingreso y la diversidad cultural. Para cumplir dichos propósitos se considera que la agenda urbana debe asumir estrategias integrales y multidimensionales (UN-Habitat, 2009: 35), las cuales resultan poco convencionales en el caso mexicano.

Entre los efectos más importantes del cambio climático destaca el estrés hídrico, debido a que la disponibilidad de agua en calidad y cantidad insuficientes representa un riesgo económico y social para la población, quien ve comprometida de manera directa su salud y alimentación; por tanto, dicha condición es un factor central en la vulnerabilidad social (CEPAL, 2009: 28).

En este sentido, el análisis del estrés hídrico implica una estrategia metodológica multidimensional que permita abordar la interconexión entre hidrología, ecología y sociedad (UNESCO, 2009: 151). En general, se considera importante mitigar los efectos en la provisión de servicios ambientales; el criterio para alcanzar dicho propósito tiene que ver precisamente con buscar el equilibrio natural en los sistemas hídricos, definidos particularmente en las cuencas (IFPRI-IWMI, 2002: 19).

Así, se considera que las cuencas funcionan como una sola unidad territorial donde se dan de manera natural toda una serie de servicios ambientales, interconectados con el ciclo hidrológico (Seckler, 1996: 6; Rijsberman y Molden, 2001: 9). En esta lógica, la instalación y el funcionamiento de las aglomeraciones humanas son factores relevantes en la modificación de las cuencas, ya que entrañan una alta concentración de población y producción económica en estas unidades territoriales (Keller et al., 2000: 5). Por tanto, el diagnóstico de los factores que modifican las cuencas es útil para comprender el estatus que guarda la disponibilidad de agua.

La instalación y el funcionamiento de las aglomeraciones humanas implican concretamente la fragmentación territorial que afecta las corrientes superficiales; la sobreextracción de fuentes subterráneas utilizada para riego y consumo humano; el cambio de las condiciones fisiográficas originales debido a la sustitución de usos del suelo, y la contaminación (Revenga et al., 2000: 15).

En este trabajo se considera que el diagnóstico del estatus en la disponibilidad de agua es una necesidad concreta del país, dada la creciente tasa de urbanización que incrementa la demanda del recurso (Conapo, 2005), así como por las dificultades inherentes de salud y alimentación. 
La producción de granos básicos, por ejemplo, muestra una balanza comercial negativa (CD, 2011), lo que hace a México un país dependiente. Como se mencionó, tanto la alimentación como la salud representan precisamente dos de los problemas más complejos a resolver en la escala mundial, y que agudizan la pobreza en la escala local. La posibilidad regional de mitigarlos representa una condición de emergencia.

Ahora bien, las posibilidades de intervención o de mitigación desde lo territorial se concentran en los elementos de la oferta potencial, porque es en estos donde las aglomeraciones humanas intervienen de manera directa. En sentido estricto, un diagnóstico del estatus en la disponibilidad de agua incluye varios elementos que se salen de los objetivos de este trabajo, es decir, el enfoque del sistema hídrico regional que implica el funcionamiento entre cuencas, transfronterizo y en conjunto; la estimación de la demanda agregada del recurso, según las particularidades locales; la descripción de los factores como la cantidad y la calidad de agua, entre otros.

Atendiendo exclusivamente a la modificación del sistema hídrico, ésta se puede analizar con base en diferentes factores (cuadro 1), sin embargo, el propósito final de esta investigación es diagnosticar solamente aquellos en los que es posible plantear esquemas de intervención a partir de las políticas territoriales, es decir, el cambio de usos del suelo por cuenca y la sobreexplotación de fuentes subterráneas.

\section{Cuadro 1 \\ Factores que modifican el sistema hídrico}

\begin{tabular}{ll}
\hline \multicolumn{1}{c}{ Factor } & \multicolumn{1}{c}{ Indicadores } \\
\hline Ríos & $\begin{array}{l}\text { Fragmentación, regulación de flujos, } \\
\text { sedimentación y retención de nutrientes }\end{array}$ \\
Fuentes subterráneas & $\begin{array}{l}\text { Sobreexplotación e intrusión salina } \\
\text { Púrdida de humedales }\end{array}$ \\
Escala de cuenca & $\begin{array}{l}\text { Cambio de usos del suelo agrícola, urbano } \\
\text { e industrial }\end{array}$ \\
\hline
\end{tabular}

Fuente: Elaboración propia con base en Revenga et al., 2000.

Por tanto, el sistema urbano de cuenca (suc) se plantea como una categoría territorial útil para fijar una escala de análisis e instrumentación de políticas, donde la oferta potencial y la demanda de agua puedan aproximarse, por la vía de la mitigación de los procesos de fragmentación territorial y por la modificación de los patrones de consumo. Con ello es posible mitigar el estrés hídrico, lo cual es un objetivo poco convencional en las políticas urbanas nacionales. 
Metodológicamente, el suc se integra con la aglomeración humana total localizada dentro de cada cuenca, incluyendo áreas urbanas consolidadas, poblaciones de menores dimensiones y el territorio no urbano (agrícola y forestal); en otras palabras, se considera una sola unidad territorial campo-ciudad, pero bajo los límites ambientalmente funcionales de la cuenca.

$\mathrm{Al}$ respecto, las políticas territoriales se pueden integrar en un programa de desarrollo del sistema urbano de cuenca (PDSuc), donde se asume que la unidad indisociable campo-ciudad queda dentro de los límites funcionales del ciclo hidrológico e integra una diversidad de actividades humanas dependientes ambientalmente.

La estructura del análisis responde al siguiente orden: $a$ ) de un total de 159 cuencas registradas por la Comisión Nacional del Agua (Conagua, 1998), se consideran 45 debido a que en ellas se genera $92.85 \%$ del PIB del país, es decir, se elige una muestra donde se concentra la mayor demanda de recursos y se reproduce un creciente estado de vulnerabilidad social; b) se identifican los sectores de la actividad económica predominantes en las cuencas de estudio para estimar la demanda efectiva, $\mathrm{y} c$ ) se estiman los factores que modifican el sistema hídrico, particularmente la extracción de agua y la sustitución de usos del suelo.

Cabe destacar que la metodología utilizada esquematiza un eje de diagnóstico no convencional porque se concentra en los factores que modifican el sistema hídrico; esto es, los patrones de extracción asociados a las aglomeraciones humanas y las principales actividades económicas. Con ello es posible analizar las políticas territoriales predominantes, pero sobre todo subrayar la importancia del recurso agua, el cual es fundamental para mitigar la vulnerabilidad de la población en términos de la alimentación y la salud. En este sentido, las estrategias para mitigar el estrés hídrico en los suc tienen diferentes planos de intervención, tantos como los factores que modifican el sistema hídrico, sin embargo se considera que desde las políticas urbanas es posible abordar dos condiciones centrales: la reducción de los procesos de fragmentación territorial ocasionados por las aglomeraciones humanas y los patrones de extracción de la sociedad, porque vienen a afectar progresivamente la oferta potencial del recurso y no son parte de la agenda urbana en México.

\section{Consideraciones sobre la adaptación al cambio climático}

En la sociedad moderna el desarrollo económico depende del consumo intensivo de combustible fósil, sin embargo, éste constituye la principal fuente de emisión de gases a la atmósfera que contribuyen al cambio 
climático o que se identifica como una muy probable causa de ello (IPCC, 2007a: 103).

La decisión política de sostener los patrones de desarrollo se sustenta en la posibilidad de abatir las desigualdades sociales. El desarrollo económico se considera el mecanismo adecuado para conseguir tal propósito, aun cuando se reconoce su potencial de afectación planetaria (вм, 2010: 234), y se estiman los costos económicos que ésta genera (CEPAL-BID, 2009: 19).

En este marco, la mitigación de los efectos en el ambiente se reduce a incorporar desarrollos tecnológicos y a optimizar la energía. Sin embargo, hay una serie de limitaciones atribuibles a la racionalidad económica predominante, mismas que no comparten un mismo nivel de atención pero constituyen aspectos coyunturales, incluidos los esquemas distributivos o, por el contrario, la modificación misma de los patrones de producción y consumo. Debido a ello, los avances en las diferentes esferas no necesariamente implican la distribución homogénea de beneficios, y tampoco la reducción de la vulnerabilidad social o ambiental.

Ahora bien, se discute que los riesgos económicos y la vulnerabilidad social están directamente asociados al cambio climático, por lo que se vuelve necesaria la incorporación de esquemas de mitigación y adecuación. Estos se refieren a la posibilidad de modificar en el corto y mediano plazos las condiciones prevalecientes de la interrelación humana o, como sociedad, con el ambiente (IPCC, 2007c: 142).

Los esquemas de adaptación y adecuación tienen un espectro de posibilidades que se han sistematizado por sector, que incluye agua, agricultura, infraestructura para asentamientos, turismo, transporte y energía. Por su relevancia para la alimentación, la salud y en general para el funcionamiento de la vida misma, se considera que la disponibilidad de agua se encuentra entre las acciones globales más relevantes (Rosegrant et al., 2002: 3). Incluso, el estado de estrés hídrico representa serias dificultades para sostener los patrones de desarrollo económico prevalecientes (UNESCO, 2006: 82).

En términos de disponibilidad de agua, la mitigación y la adaptación al cambio climático se sugiere en diferentes escalas de intervención. En lo local se propone potenciar la captura de agua pluvial, técnicas de almacenamiento y conservación, reutilización, desalación, eficiencia de uso y de la irrigación; mientras que en lo regional se considera la incorporación de políticas nacionales sobre el agua, gestión integrada de los recursos hídricos y gestión de fenómenos peligrosos relacionados con el agua (IPCC, 2007b: 57).

Por su potencial regional, destaca el manejo integrado de recursos hídricos (MIRH), el cual se considera "una forma de desarrollo y adminis- 
tración de agua, suelo y recursos, útil para maximizar los beneficios económicos y sociales en forma equitativa, sin comprometer la sustentabilidad de los ecosistemas" (GWP-INBO, 2009: 18). El MIRH se considera en la escala territorial de cuenca y acuífero.

De hecho, entre los consensos derivados del quinto Foro Mundial del Agua (2009) se encuentra precisamente "el manejo y la protección de los recursos y sus sistemas de abastecimiento para alcanzar las necesidades humanas y ambientales" (wwc, 2009: 22). Cabe destacar que la instrumentación de estrategias de adaptación con base en el PMIRH ha encontrado serias dificultades en términos administrativos, políticos y de información básica, sobre todo en los países subdesarrollados (UN-Water, 2008: 16).

\section{El sistema urbano de cuenca como esquema de manejo integrado de recursos hídricos}

En general, el MIRH en la escala de cuenca responde al objetivo de gestionar el aprovechamiento y la administración de los recursos de forma sistémica. Para que esto se cumpla se requiere que se consideren los intereses de los diversos usos y usuarios, la integración de aspectos centrales como cantidad y calidad, la administración de la oferta y la demanda, la planeación de los diferentes componentes del agua o de las distintas fases del ciclo hidrológico y la consideración del desarrollo económico, social y ambiental (Dourojeanni et al., 2002: 18).

No obstante, la perspectiva predominante en los países subdesarrollados es el aprovechamiento continuo e ininterrumpido del recurso, donde se pondera la actividad económica. Además, en los casos donde existe una instrumentación de políticas, se observa la falta de coordinación y de transversalidad que resultan en la competencia e intervención de múltiples entidades administrativas, agudizando los conflictos del sector. En este sentido, la característica común en la gestión del agua en los países subdesarrollados es la visión sectorial para resolver demandas específicas y no las condiciones del recurso (Dourojeanni y Jouravlev, 1999: 50).

Por tanto, la escala de cuenca se acepta como unidad de gestión en el MIRH. Aunque se reconocen diversas dificultades operativas y analíticas para su integración; incluso la generación e instrumentación de políticas sigue en proceso de construcción (Garduño et al., 2006: 4; GWsp, 2005: 46; Smakhtin et al., 2004: 2; Molle y Hoanh, 2009: 13; IвRD/вм, 2010: 39; Hirji y Davis, 2009: 5).

En los casos donde existen avances, estos alcanzan diferentes niveles de desarrollo y consolidación, normalmente asociados al grado de desarrollo de los países. Los conflictos más comunes en los países subdesarrollados tienen que ver con la falta de coordinación entre diferentes sectores 
o, por el contrario, se genera una competencia entre ellos para intervenir en la administración del recurso (Molle, 2006: 23).

En particular, México cuenta con organismos encargados de administrar las aguas nacionales en las escalas de país y regional, entre ellas la Conagua y 13 gerencias regionales u organismos de cuenca, los cuales tienen como objetivo: "La gestión integrada de los recursos hídricos, superficiales y del subsuelo, a partir de las cuencas hidrológicas en el territorio nacional, como prioridad y asunto de seguridad nacional" (DOF, 2008). Para desempeñar esta función, el país se subdivide en 13 Regiones Hidrológico-Administrativas formadas por agrupaciones de cuencas que se consideran unidades básicas de gestión de los recursos hídricos (DOF, 1997).

La delimitación de las regiones hidrológicas responde exclusivamente a criterios administrativos, por lo que estos parcializan las posibilidades de análisis e intervención, además de reducir el potencial multidimensional de la gestión de los recursos hídricos. En consecuencia, las gerencias regionales tendrían que operar bajo el criterio de cuenca para cumplir dicho propósito. Además, en el caso mexicano existen tres versiones en la delimitación de éstas, a saber: el Instituto Nacional de Estadística, Geografía e Informática, el Instituto Nacional de Ecología y la Comisión Nacional del Agua (Conagua, 1998; Maderey y Torres, 1990).

En este trabajo el suc se tiene como una unidad básica para la gestión de los recursos hídricos. A reserva de que la operación de las gerencias regionales puede considerarse de sistema urbano de cuenca, la ventaja de tal estrategia consiste en ofrecer un diagnóstico más certero de las condiciones de la gestión en el nivel de cuenca.

No es el objetivo en este trabajo analizar los criterios técnicos de la delimitación de las cuencas, por lo que se asume aquella versión donde se tiene un nivel mayor de desagregación del territorio (Conagua, 1998), lo que permite estudiar con mayor precisión los factores de aprovechamiento y afectación del sistema hídrico.

También se asume que la mayor transformación territorial la ocasiona el sistema urbano nacional, y que las políticas urbanas no registran claramente la emergencia del manejo integrado de recursos hídricos. Por ello la delimitación de cuenca se ensambla al proceso urbano en su territorio, para segmentar los efectos pero también para diversificar los esquemas de atención que finalmente son de adecuación y mitigación para un contexto de emergencia de cambio climático.

\section{Sistema urbano de cuenca}

Este sistema se plantea como una unidad básica para el manejo integrado de los recursos hídricos. En la versión de la Conagua (1998) se identifican 1,738 cuencas, incluidas 159 localizadas en la parte continental y el 


\section{Mapa I \\ Sistemas urbanos de cuenca \\ (muestra)}

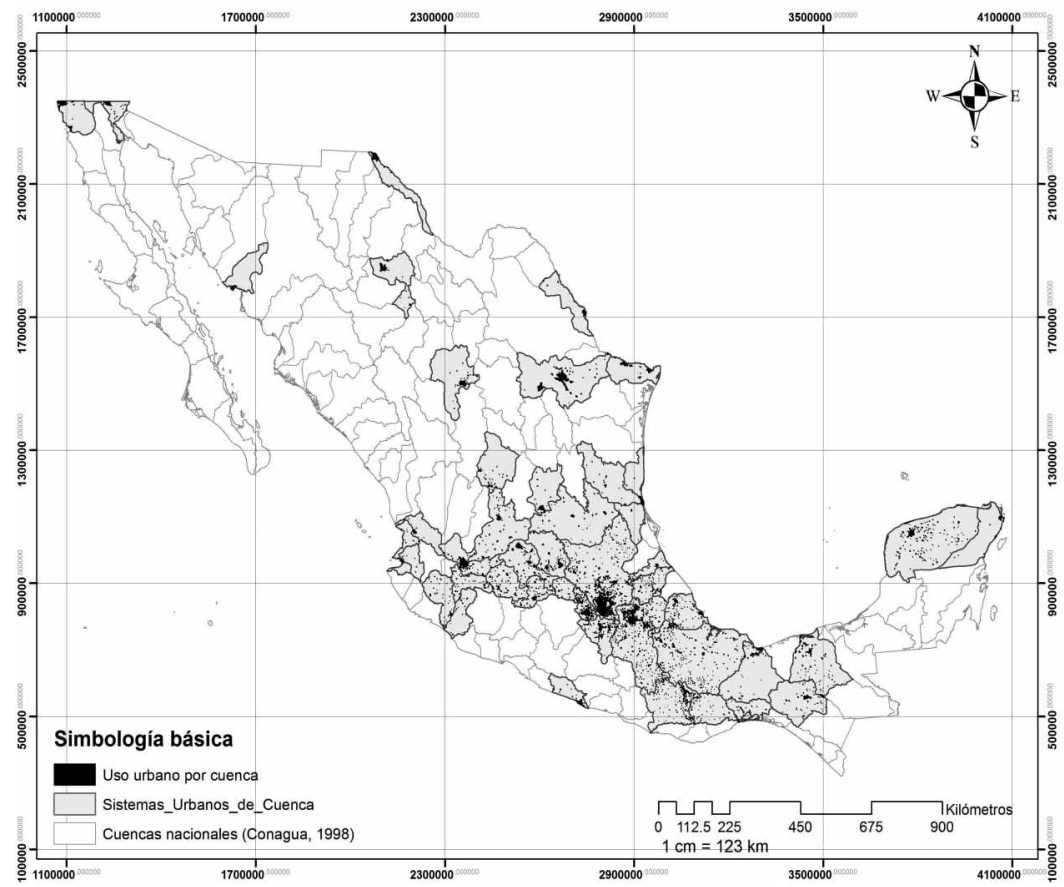

Fuente: Elaboración propia con base en Conabio (1997) y Conagua (1998).

resto se distribuyen en islas. Se asume que la instalación y funcionamiento de las aglomeraciones humanas son factores centrales para la modificación del sistema hídrico, por lo que estos se analizan en términos de los cambios de usos del suelo y la sobreexplotación de fuentes subterráneas en una muestra de 45 cuencas (mapa I).

De forma agregada, los suc se componen por las 56 zonas metropolitanas reconocidas por el Conapo (2005) y por 144,207 localidades menores; estas últimas representan $50 \%$ de la cantidad total registrada en 2005 (cuadro 2).

El análisis del cambio de uso del suelo y de la sobreexplotación de fuentes subterráneas permite estimar las condiciones de la oferta potencial del recurso, es decir, la capacidad para sostener el volumen disponible para su uso y aprovechamiento. Derivado de ello, las políticas territoriales significan una posibilidad de adecuación de las aglomeraciones humanas ante las contingencias del estrés hídrico, o en su defecto esquematizar regionalmente el MIRH. 


\section{Cuadro 2}

Composición de sistemas urbanos de cuenca

\begin{tabular}{|c|c|c|c|}
\hline Núm. & Cuenca & Zona metropolitana & Localidades \\
\hline 1 & Bacanora-Mejorada & Tijuana & 2,330 \\
\hline 2 & $\begin{array}{l}\text { Arroyo Tijuana-Arroyo } \\
\text { de Maneadero }\end{array}$ & Mexicali & 2,357 \\
\hline 3 & Río Bravo-Ciudad Juárez & Juárez & 342 \\
\hline 4 & Río Matape & Guaymas & 994 \\
\hline 5 & Río Conchos-Presa el Granero & Chihuahua & 1,998 \\
\hline 6 & Río Bravo-Nuevo Laredo & Nuevo Laredo & 1,147 \\
\hline \multirow[t]{2}{*}{7} & Río Nazas-Torreón & La Laguna & 2,217 \\
\hline & & Matamoros & \\
\hline \multirow[t]{2}{*}{8} & Río Bravo-San Juan & Monterrey & 6,805 \\
\hline & & Saltillo & \\
\hline 9 & Río Bravo-Matamoros-Reynosa & Reynosa-Río Bravo & 3,231 \\
\hline 10 & Fresnillos-Yesca & Zacatecas-Guadalupe & 1,600 \\
\hline 11 & Río Tamesí & Tampico & 3,153 \\
\hline 12 & Lago de San Andrés-Lago Morales & Tampico & 1,304 \\
\hline 13 & San Luis Potosí & San Luis Potosí-Soledad & 1,309 \\
\hline 14 & Río Tamuín & Río Verde-Ciudad Fernández & 5,484 \\
\hline 15 & Río Verde Grande & Aguascalientes & 7,033 \\
\hline 16 & Río Pánuco & Tampico & 2,821 \\
\hline 17 & Santiago-Aguamilpa & Tepic & 1,113 \\
\hline \multirow[t]{4}{*}{18} & Río Moctezuma & Valle de México & 12,595 \\
\hline & & Pachuca & \\
\hline & & Tulancingo & \\
\hline & & Tula & \\
\hline 19 & Río Huicicila-San Blas & Tepic & 626 \\
\hline 20 & Yucatán & Mérida & 9,395 \\
\hline 21 & Quintana Roo & Cancún & 1,496 \\
\hline 22 & Río Laja & Querétaro & 4,897 \\
\hline \multirow[t]{2}{*}{23} & Río Santiago Guadalajara & Guadalajara & 3,362 \\
\hline & & Ocotlán & \\
\hline \multirow[t]{2}{*}{24} & Río Lerma-Salamanca & León & 5,404 \\
\hline & & San Francisco del Rincón & \\
\hline 25 & Río Ameca-Ixtapa & Puerto Vallarta & 697 \\
\hline 26 & Río Cazones & Poza Rica & 1,627 \\
\hline \multirow[t]{2}{*}{27} & Río Lerma-Chapala & Zamora-Jacona & 2,178 \\
\hline & & La Piedad-Pénjamo & \\
\hline
\end{tabular}


Continuación Cuadro 2...

\begin{tabular}{|c|c|c|c|}
\hline Núm. & Cuenca & Zona metropolitana & Localidades \\
\hline 28 & Río Cuale-Pitillal & Puerto Vallarta & 232 \\
\hline 29 & Lago Chapala & Guadalajara & 1,394 \\
\hline 30 & Río Tecolutla & Poza Rica & 2,574 \\
\hline 31 & Río Armería & Colima-Villa de Álvarez & 2,365 \\
\hline \multirow[t]{2}{*}{32} & \multirow{2}{*}{$\begin{array}{l}\text { Lago de Pátzcuaro-Cuitzeo y Lago } \\
\text { de Yuriria }\end{array}$} & Morelia & \multirow[t]{2}{*}{1,264} \\
\hline & & Moroleón-Uriangato & \\
\hline 33 & Río Lerma-Toluca & Toluca & 1,784 \\
\hline 34 & Río Coahuayana & Tecomán & 2,519 \\
\hline \multirow[t]{2}{*}{35} & \multirow[t]{2}{*}{ Río Jamapa y otros } & Veracruz & \multirow[t]{2}{*}{4,161} \\
\hline & & Xalapa & \\
\hline \multirow[t]{2}{*}{36} & \multirow[t]{2}{*}{ Río Atoyac-A } & Puebla-Tlaxcala & \multirow[t]{2}{*}{6,226} \\
\hline & & Tlaxcala-Apizaco & \\
\hline \multirow[t]{2}{*}{37} & \multirow[t]{2}{*}{ Río Grande de Amacuzac } & Cuernavaca & \multirow[t]{2}{*}{2,270} \\
\hline & & Cuautla & \\
\hline \multirow[t]{4}{*}{38} & \multirow[t]{4}{*}{ Río Papaloapan } & Orizaba & \multirow[t]{4}{*}{11,081} \\
\hline & & Córdoba & \\
\hline & & Tehuacán & \\
\hline & & Acayucan & \\
\hline 39 & Río Grijalva-Villa Hermosa & Villahermosa & 4,924 \\
\hline \multirow[t]{2}{*}{40} & \multirow[t]{2}{*}{ Río Coatzacoalcos } & Minatitlán & \multirow[t]{2}{*}{3,584} \\
\hline & & Coatzacoalcos & \\
\hline 41 & Río Atoyac y otros & Acapulco & 881 \\
\hline 42 & Río Atoyac-B & Oaxaca & 2,932 \\
\hline 43 & Río Grijalva-Tuxtla Gutiérrez & Tuxtla Gutiérrez & 7,204 \\
\hline 44 & Río Tehuantepec & Tehuantepec & 605 \\
\hline \multirow[t]{2}{*}{45} & \multirow[t]{2}{*}{ Laguna Superior e Inferior } & \multirow[t]{2}{*}{ Tehuantepec } & 692 \\
\hline & & & 144,207 \\
\hline
\end{tabular}

Fuente: Elaboración propia con base en Conabio (1997), Conagua (1998) e INEGI (2005).

Ahora bien, las actividades económicas en regiones específicas suponen un aprovechamiento ambiental que es cualitativa y cuantitativamente diferencial. En primera instancia, dicho aprovechamiento está asociado a la intensidad de la actividad económica, misma que se estima convencionalmente con la participación porcentual a la renta nacional o en términos del PIB per cápita.

\subsection{Distribución regional de la actividad económica}

El 92.85\% del pib se genera en 45 de 159 cuencas, en un territorio equivalente a $32.05 \%$ del total nacional (mapa ii). La participación regional 
al рів permite analizar la disparidad en términos de la capacidad productiva, y al mismo tiempo los desequilibrios locales y extraterritoriales ocasionados por la alta concentración de la demanda de recursos.

En general, la actividad económica refleja diferentes patrones de concentración espacial y de especialización que son determinantes de la demanda de recursos. Los suC registran 1.5 zonas metropolitanas en promedio, sin embargo, $14.32 \%$ del territorio nacional (Río Moctezuma y Río Papaloapan) registra la mayor aglomeración humana del país, incluidas ocho zonas metropolitanas ${ }^{1}$ y 23,676 localidades menores. En contraste, el menor rango es Río Cuale-Pitillal, donde solamente existe una zona metropolitana (Puerto Vallarta) y 232 localidades. Por tanto, los umbrales del uso y aprovechamiento de agua están condicionados por la escala de las aglomeraciones. Al mismo tiempo, la mitigación de tales desequilibrios debe ser objeto de políticas regionales, dados los límites de la capacidad de carga del ecosistema.

Por otro lado, la capacidad productiva y la especialización económica generan patrones diferenciales en la modificación del sistema hídrico. La participación estatal al PIB nacional es de 3.32\% en promedio, sin embargo Río Moctezuma, Río Lerma Toluca, Río Bravo San Juan, Río Santiago Guadalajara, Río Ameca Ixtapa, Río Cuale Pitillal, Lago Chapala y Río Coahuayana aportan dos veces o más (mapa II), justo donde se localizan las zonas metropolitanas con más de un millón de habitantes.

El 67.04\% del PIB proviene del sector terciario, el cual predomina en $10.31 \%$ del territorio nacional, en 13 cuencas (figura I). En la práctica, una proporción menor del país concentra al sector más productivo, el cual se localiza en una franja que va del occidente al Golfo de México entre Guadalajara, Distrito Federal y Veracruz. Por su parte, el sector secundario es mayor en 24 cuencas; $^{2}$ destaca el subsector de construcción en Río Moctezuma y la industria manufacturera en Río Lerma Toluca y Río Bravo-San Juan. En ambos casos, la modificación del sistema hídrico combina la sobreextracción del recurso como insumo para el funcionamiento industrial y urbano, así como la fragmentación territorial para la instalación del sector inmobiliario.

Finalmente, la mayor participación del sector primario se observa en 16 cuencas, localizadas sobre todo en el centro occidente y el Golfo de México, lo cual supone una extracción de agua particularmente intensiva, ya que este sector de actividad es el que consume el mayor volumen $(76.8 \%$ del total) en el ámbito nacional (Conagua, 2010: 61).

\footnotetext{
${ }^{1}$ Valle de México, Pachuca, Tulancingo, Tula; Orizaba, Córdoba, Tehuacán, Acayucan, respectivamente.

${ }^{2}$ Por subsector, la construcción sobresale en Río Moctezuma, y la industria manufacturera en Río Lerma Toluca y Río Bravo-San Juan.
} 


\section{Mapa II}

Rangos de participación al PIB nacional por SUC

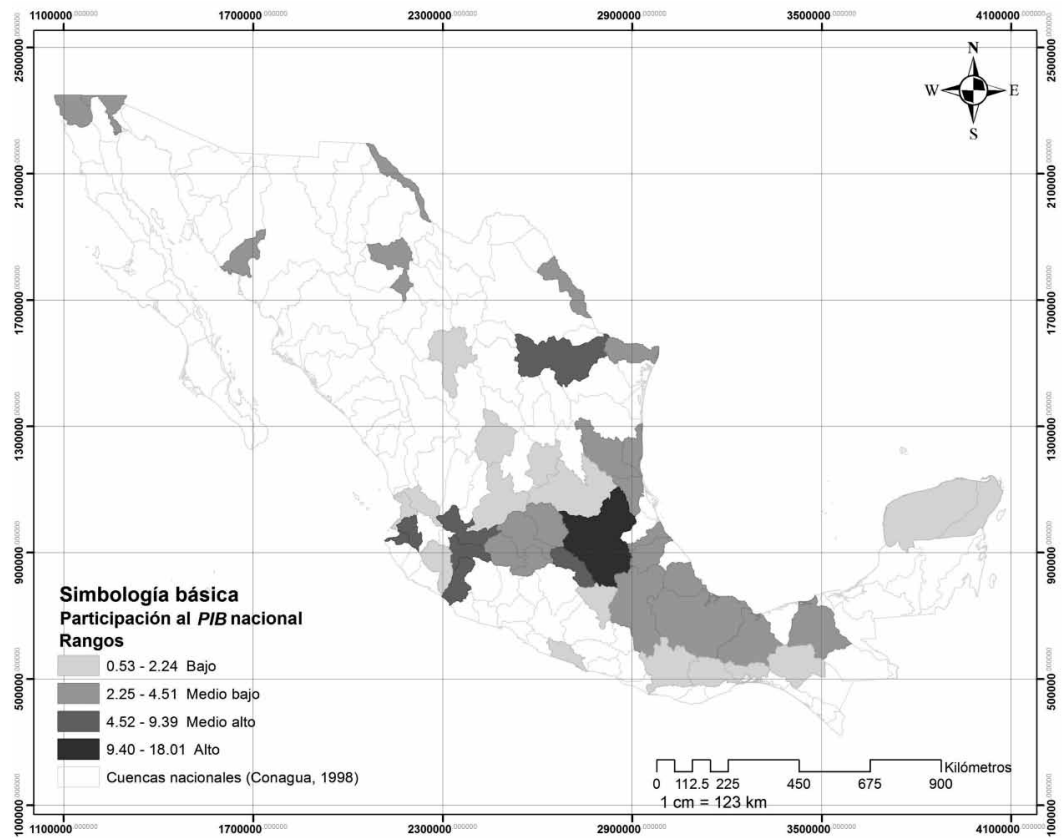

Fuente: Elaboración propia con base en Conabio (1997), Conagua (1998) e INEGI (2005).

Figura I

Participación al PIB nacional por sistema urbano de cuenca

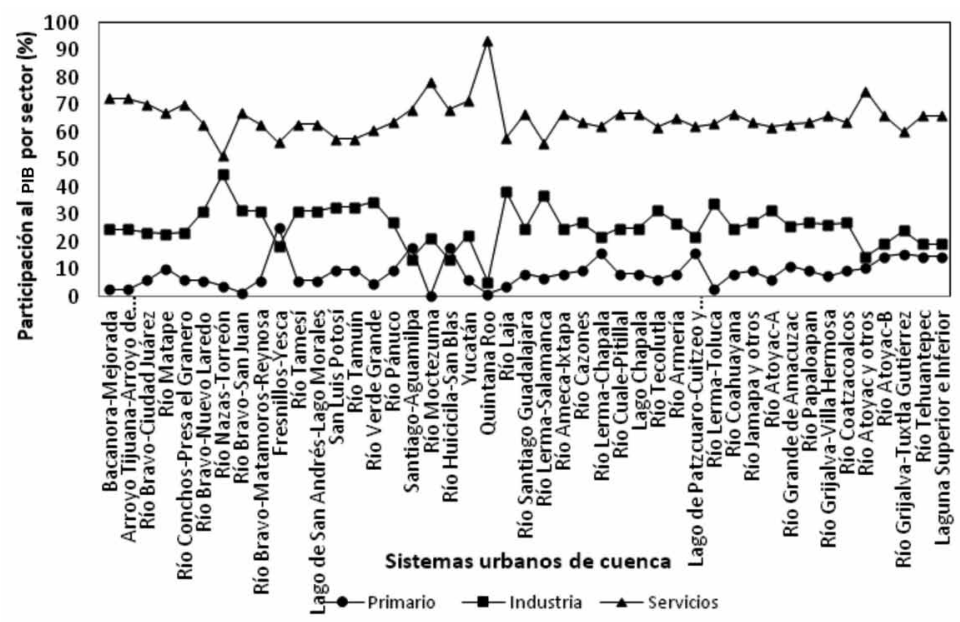

Fuente: Elaboración propia con base en Conabio (1997), Conagua (1998) e INEGI (2005). 
La distribución territorial de las actividades económicas refleja condiciones diferenciales en el aprovechamiento y la modificación del sistema hídrico. El caso más representativo es el suc Río Moctezuma, donde se localiza el Distrito Federal. En conjunto, la Zona Metropolitana del Valle de México (zmvm) es responsable de $18.01 \%$ del pib. Esta capacidad productiva requiere una intensa extracción de agua que ha llevado a agotar las fuentes locales y a limitar la infiltración local. En consecuencia, para sostener su funcionamiento importa $30 \%$ del volumen utilizado desde una cuenca localizada a $100 \mathrm{~km}$ de distancia (Legorreta, 2006: 102), donde se manifiestan los límites derivados de la interrelación entre la actividad económica y el ambiente. En este eje de análisis, los suc que aportan dos veces o más al PIB pueden ser vulnerables en la medida en que su sistema hídrico no responda a un manejo integrado, y dada la evidencia, a mayor producción económica mayor dependencia ambiental.

\subsection{Producto interno bruto per cápita}

Los patrones de desarrollo se sustentan en la posibilidad de abatir las desigualdades sociales. La segunda muestra de disparidad regional tiene que ver con la distribución del рів per cápita, el cual se utiliza para describir los avances sociales de la actividad económica, así como para validar las ventajas económicas de las aglomeraciones humanas.

En este sentido, el país registra un PIB per cápita promedio de 7,595 dólares al año, que corresponde a un nivel de desarrollo humano alto, según el Programa de Naciones Unidas para el Desarrollo (PNUD, 2009). No obstante, $92.91 \%$ de los municipios se encuentra por debajo de este registro; tal disparidad tiene un rango que va de 35,595 a 224 dólares al año, donde sobresale $66.68 \%$ del territorio nacional con un promedio de 116 pesos, cantidad similar a países africanos con el menor índice de desarrollo humano.

En el contexto regional, existe una clara concentración de los más altos registros del PIB per cápita: en el centro-norte del país sobresalen Río Moctezuma, Río Verde San Juan, Río Verde Grande, Río Laja, Río Nazas Torreón, Río Bravo Ciudad Juárez y Bacanora Mejorada; mientras que en el sur-sureste ningún suc alcanza el promedio nacional, excepto Quintana Roo (mapa III). Aun cuando solamente 10 de 45 suc se encuentran en los rangos bajo y medio bajo de PIB per cápita, existen otros elementos para subrayar las disparidades, como la proporción de población económicamente activa con el nivel de ingreso más bajo ${ }^{3}$ o el porcentaje vivienda con algún nivel de hacinamiento, ${ }^{4}$ cuya proporción y distribución es relativamente homogénea en todos los suc.

${ }^{3}$ El $50.99 \%$ de la población ocupada tiene un ingreso de hasta dos salarios mínimos.

${ }^{4}$ El $45.94 \%$ de la vivienda registrada. 


\section{Mapa III \\ PIB per cápita por SUC}

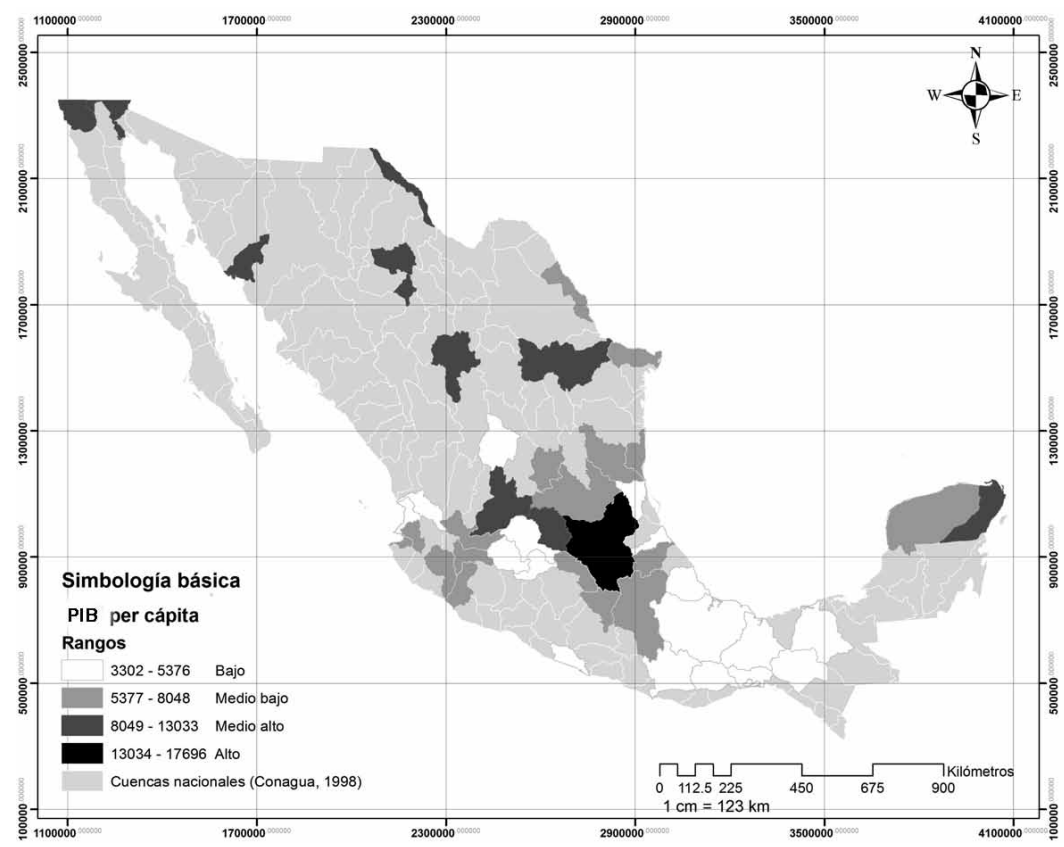

Fuente: Elaboración propia con base en Conabio (1997), Conagua (1998) e INEGI (2005).

Con base en el PIB se observa una alta concentración de la capacidad productiva en algunos suc del centro, occidente y norte del país; Río Moctezuma en particular es responsable de una quinta parte en el ámbito nacional; sin embargo, internamente registra una fuerte concentración de los niveles de ingreso más bajos, así como una serie de dificultades para proveerse del volumen de agua necesario. Como se comentó, la alta concentración de población y de capacidad productiva también se expresan en disparidad social determinada por la pobreza, así como vulnerabilidad ambiental.

\section{Modificación del sistema hídrico}

La modificación del sistema hídrico está condicionada por el tipo de actividad económica y sus patrones de concentración territorial. La evidencia demuestra que la mayor producción se concentra en una franja que va del occidente al Golfo de México -entre Guadalajara, Distrito Federal y Veracruz-, y que el sector terciario es responsable de la mayor 
aportación al PIB nacional, aun cuando el sector secundario es predominante en una amplia zona del centro y el occidente.

En términos ambientales, la información parece indicar que a mayor concentración de capacidad productiva más vulnerabilidad ambiental, por lo menos en términos de la disponibilidad de agua. Se evidencia además que la alta capacidad productiva no implica un distribución homogénea de beneficios, dada la distribución del piв per cápita o los niveles de ingreso y hacinamiento.

Además, la consolidación y el funcionamiento del espacio urbano se identifican como causas principales del cambio de usos del suelo por cuenca y de la sobreexplotación de fuentes subterráneas. En este sentido, las políticas territoriales tienen pendientes importantes entre los que destacan: el replanteamiento del proyecto productivo de país con base en el aprovechamiento racional del sistema natural, es decir, con un esquema de manejo integrado de recursos y al mismo tiempo el planteamiento de estrategias de mitigación para el escenario del cambio climático. En ambos casos resulta implícito plantear la redistribución de las condiciones económicas, así como la atención al estrés hídrico, es decir, las condiciones generales de vulnerabilidad y disparidad regional.

\subsection{Sobreextracción de fuentes subterráneas}

En términos del MIRH, se considera necesaria la administración del agua y la regulación de la modificación de los usos del suelo. Por tanto, determinar las estrategias posibles de administración resulta pertinente, analizar el grado de presión en el recurso, así como su consecuente estado de disponibilidad per cápita.

En primera instancia, el grado de presión implica estimar el porcentaje de agua recuperado respecto al volumen extraído, ${ }^{5}$ de acuerdo con los patrones de consumo y fragmentación territorial predominantes. En este sentido, las condiciones regionales determinan la disponibilidad per cápita, y según los rangos de aprovisionamiento es posible estimar las dificultades que estos implican para la vida humana, es decir, el nivel de estrés hídrico al que está sometida la sociedad.

Según Revenga et al. (2000), la disponibilidad per cápita de agua en los rangos 500, 1,000 y 1,700 $\mathrm{m}^{3} /$ persona/año implican efectos directos en el funcionamiento de la sociedad, ${ }^{6}$ y representan el mayor estado de estrés hídrico.

\footnotetext{
${ }^{5} \mathrm{Tal}$ referencia se considera como el volumen de recarga media anual (Conagua, 2010: 18). En este trabajo se toman las estimaciones hechas para el país en el año 2010.

${ }^{6}$ a) Menos de $500 \mathrm{~m}^{3} /$ persona/año, escasez; b) 500 a 1,000, problemas crónicos; c) 1,000 a 1,700, problemas severos temporales, y d) 1,700 a 4,000, nivel óptimo.
} 


\section{Mapa IV}

\section{Disponibilidad de agua, según suc}

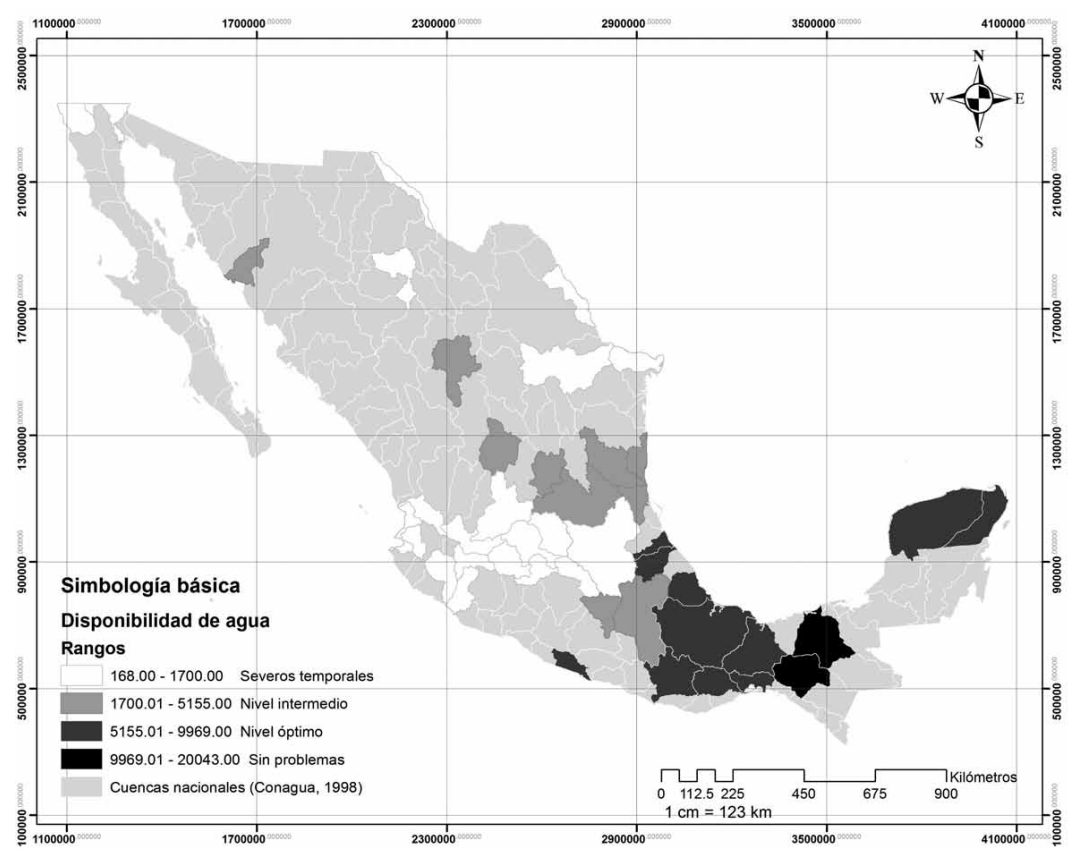

Fuente: Elaboración propia con base en Conabio (1997), Conagua (1998, 2010), Sedesol (2006), e IF, PRI-IWMI (2002).

Atendiendo a estos rangos de disponibilidad, se observa que la región del país que más aporta al PIB se encuentra por debajo de los $1,700 \mathrm{~m}^{3 /}$ persona/año. Dicho de otra forma, la región con mayor capacidad productiva refleja el nivel de estrés hídrico más apremiante, considerado entre escasez y problemas severos temporales, que significan efectos adversos para la población (mapa Iv).

La estimación en el grado de presión del recurso se complementa con los indicadores de fragmentación territorial que reducen progresivamente el equilibrio natural de recuperación. En tal escenario, el potencial productivo de los suc es vulnerable en la medida en que se registren altas tasas de sustitución de usos del suelo. Considerando solamente la tasa de crecimiento urbano, las regiones centro, occidente y norte del país correspondiente a los suc Río Moctezuma, Río Lerma Toluca, Río Bravo San Juan, Río Santiago Guadalajara, Río Ameca Ixtapa, Río Cuale Pitillal, Lago Chapala y Río Coahuayana se encuentran en dicha situación. 


\section{Figura II \\ Usos de suelo con mayor tasa de sustitución (1994-2005)}

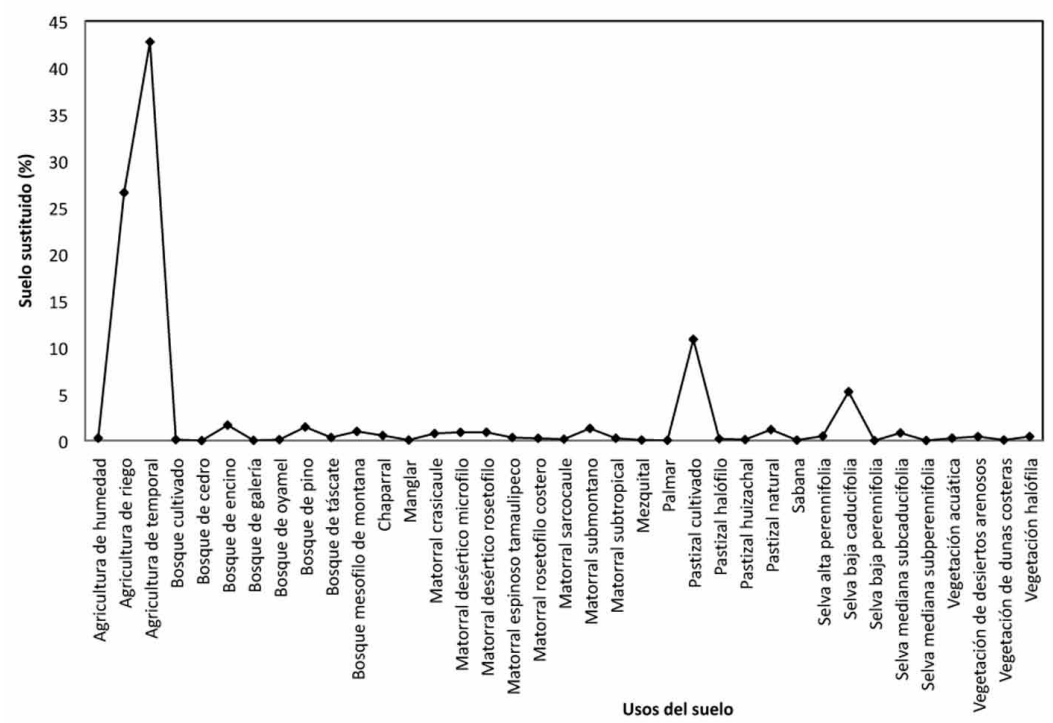

Fuente: Elaboración propia con base en Conabio (1997), Conagua (1998), INEGI-INE (1996).

\subsection{Fragmentación territorial}

El segundo elemento que modifica el sistema hídrico es el cambio de usos del suelo, debido a que la fragmentación territorial interviene en el equilibrio del sistema natural, así como en sus procesos internos de mantenimiento y recuperación. En el caso particular del servicio ambiental de disponibilidad de agua, ésta se ve disminuida.

En este contexto, la fragmentación territorial se puede estimar con base en los tipos de uso del suelo con mayor tasa de sustitución por cuenca, y por el área total afectada por el crecimiento urbano; ambos casos son útiles para determinar aspectos regionales de la política territorial. Entre 1990 y 2010 , los cuatro tipos de uso del suelo con mayor tasa de sustitución fueron el agrícola de temporal (42.75\%), el agrícola de riego (26.58\%), el pastizal cultivado (10.85\%), la selva baja caducifolia (5.24\%) y el bosque (4.69\%) (figura II).

En primera instancia, la fragmentación territorial afecta la producción de alimento, dado que $69.33 \%$ del área total sustitutida proviene de suelo agrícola. De la misma forma, con la pérdida de selva baja caducifolia se reduce una de las principales fuentes de biodiversidad nacional. Sin embargo, cabe subrayar que el cambio de usos del suelo en tanto determinante de la producción de alimento y de la disponibilidad de agua, no es 
objeto de política territorial alguna, por el contrario, el principal proveedor de suelo urbanizable de los programas urbanos resulta ser el agrícola.

Entre los factores más relevantes en la sustitución de usos del suelo está la intensidad del crecimiento urbano. Como resultado de este proceso regional, el suelo urbano ha ocupado $11,495.62 \mathrm{~km}^{2}$, lo que representa que una proporción menor del territorio nacional tiene la mayor capacidad productiva, responsable de $92.85 \%$ del PIB. Resulta igualmente relevante que en menos de $1 \%$ del territorio se provea la mayor renta nacional, y más aún, que ésta se sujete a condiciones de vulnerabilidad ambiental determinadas por la reducción de la disponibilidad de agua y de la producción agrícola, mismos que son centrales para la salud y la alimentación, respectivamente.

Ahora bien, los suc que registran la mayor área de usos del suelo sustituida por el crecimiento urbano son Río Moctezuma $\left(1,976 \mathrm{~km}^{2}\right)$, Río Atoyac-A (1,708), Río Bravo San Juan (661), Río Lerma Toluca (685), Río Papaloapan (754) y Yucatán $\left(584 \mathrm{~km}^{2}\right)$, donde se localizan las zonas metropolitanas del Valle de México (Distrito Federal, Puebla, Toluca, Pachuca, Tlaxcala), Monterrey, Veracruz y Yucatán.

Si bien la capacidad de carga de cada cuenca presenta límites distintos, la proporción de usos del suelo sustituida respecto de su área total refleja consistentemente desequilibrios que permiten estimar que los mayores rangos de fragmentación territorial también presentan más vulnerabilidad, en este caso para la disponibilidad de agua y la producción de alimentos. La evidencia demuestra que los suc con mayor proporción de suelo sustituido son Río Lerma Toluca (8.74\%), Río Atoyac-A (5.40), Río Grande Amacuzac (5.08) y Río Moctezuma ((4.92\%) (figura III).

Aunque en todos los casos se observan diferentes niveles de deterioro ambiental, sobresale Río Moctezuma, donde la fragmentación alcanza cerca de 5\% del área total de la cuenca, concentrando las más importantes deseconomías de escala, asociadas a una quinta parte del PIB. Por tanto, la interrelación entre la participación económica y los rangos permisibles de fragmentación territorial tiene que ser estudiada si se pretende conservar el potencial económico de las regiones.

\section{Consideraciones finales}

En la sociedad moderna se ha sostenido un patrón intensivo de actividad económica, que en un periodo relativamente corto ha contribuido al deterioro del sistema natural. Tal deterioro se expresa en diferentes indicadores, e incluso se considera como un problema sistémico que incluye todas las esferas de la vida. De esta forma, el cambio climático se ha convertido en un eje central para la definición de políticas públicas, 


\section{Figura III \\ Área sustituida por SUC \\ (porcentaje de cubierta original estimada para 1994)}

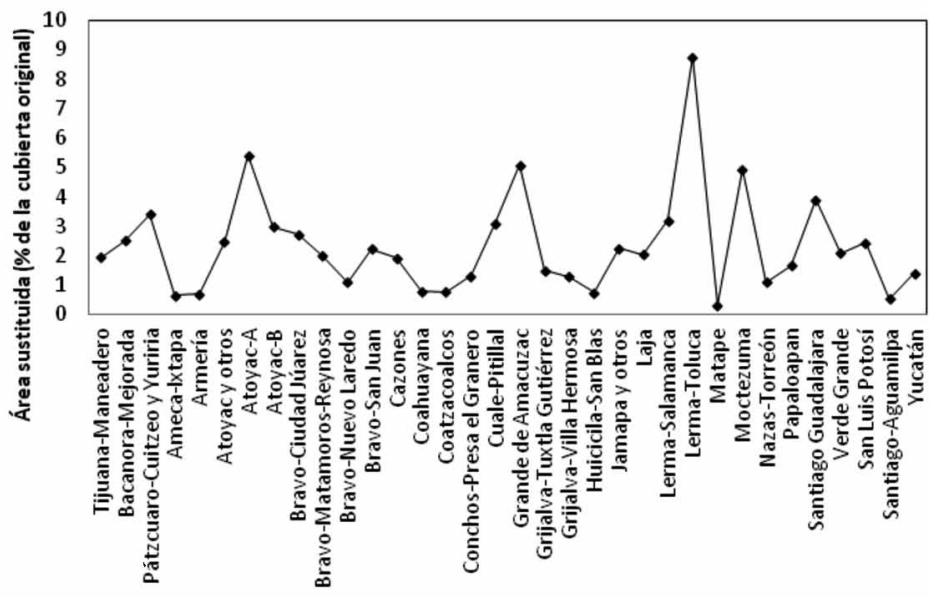

Fuente: Elaboración propia con base en Conabio (1997), Conagua (1998) e INEGI (1996).

donde se subraya la necesidad de modificar la interrelación humana con el ambiente.

A reserva de validar que la actividad humana es responsable o no del cambio climático, existe evidencia económica y social que demuestra la necesidad de mitigar los efectos adversos de éste, ya sea por los eventos naturales extremos o por la falta de agua, por ejemplo. La estrategia económica predominante ha sido buscar mecanismos de mercado para reducir costos derivados del deterioro ambiental, y en menor medida, modificar la racionalidad que lo origina.

En este contexto, el sistema urbano mundial se considera un responsable importante del cambio climático, debido a su alta concentración de actividad económica y población, pero al mismo tiempo, se encuentra que éste es un sector relevante para mitigarlo. En el último informe del Panel Intergubernamental para el Cambio Climático se menciona que las estrategias para abordarlo pueden ser de mitigación y adecuación, ambas en el escenario de una agudización de sus efectos en el corto y mediano plazos.

En este trabajo se asume dicha hipótesis para el caso particular del agua, ya que existen múltiples evidencias de que ésta es un factor que interviene definitivamente en aspectos económicos, así como en la alimentación y la salud de la población, es decir, en sus condiciones inmediatas de vida. 
Entre las estrategias técnicas y políticas que han sido desarrolladas para la mitigación de los efectos en el agua, sobresale el manejo integrado de recursos hídricos, el cual se propone como un esquema de administración y gestión para unidades territoriales funcionales, en este caso las cuencas. La instrumentación del MIRH ha tenido efectos positivos en diferentes países, pero no se ha logrado consolidar, es una estrategia en construcción. Para el caso mexicano, incluso los organismos de cuenca tienen como propósito alcanzar esta forma de manejo del agua, sin embargo, estos se caracterizan porque responden a límites administrativos y no funcionales, lo cual matiza sus posibles ventajas. Muestra de ello es que la mayor parte de las regiones hidrológico-administrativas registran una presión intensa sobre el recurso; la administración no se ha consolidado.

Ahora bien, el sistema urbano de cuenca se propone como un esquema para el MIRH para el caso mexicano. Sin embargo, este esquema permite subrayar dos condiciones estructurales: las políticas urbanas excluyen la posibilidad de mitigación o adecuación al cambio climático, aun cuando las particularidades regionales lo hacen necesario, y al mismo tiempo, la desatención sistémica de las disparidades regionales proyecta un estado creciente de vulnerabilidad económica y social.

En este sentido, se observa una alta concentración de la producción económica en fragmentos menores del territorio, sometiendo a una disparidad regional al resto del país. Además, la sobreextracción del recurso y la fragmentación territorial representan un importante riesgo para la viabilidad económica y social de las ciudades; la revisión de la falta de disponibilidad de agua por ejemplo, muestra problemas concretos para la actividad económica en la región más productiva, así como para el desarrollo del sector agrícola.

Por la dimensión del documento no es posible abordar aspectos como la calidad y cantidad de agua con un nivel de desagregación mayor, y tampoco el funcionamiento interregional de cuencas. Los dos factores representan una complejidad que tiene que ser analizada para completar este análisis. Sin embargo, el estudio de las actividades económicas respecto a la disponibilidad de agua permite subrayar la vulnerabilidad de que es objeto el sistema urbano nacional, así como la base económica del país de la que depende directamente.

En este contexto, la integración de políticas de mitigación y adecuación para el cambio climático se hace necesaria. En el consenso global existe la propuesta de incorporar la recuperación de agua pluvial, el manejo racional del recurso y la reducción de los cambios de uso del suelo, entre los más importantes. En México se enfrentan condiciones apremiantes, por lo que se debe explorar la posibilidad de incorporar dichas estrategias; el país muestra una alta dependencia económica de una región frágil 
ambientalmente, la capacidad regional para producir alimentos es muy baja y la disparidad económica de la sociedad parece no haber recogido las ventajas del desarrollo urbano. En todos los casos, el sistema urbano de cuenca sólo considera una posible estrategia de adecuación, pero no la agota, en la medida en que el MIRH tiene múltiples factores de los que no se da cuenta en el documento.

\section{Bibliografía}

Altvater, Elmar y Birgit Manhkopf (2002), Las limitaciones de la globalización, Siglo XXI, México.

Asuad, Normand, Luis Quintana y Roberto Ramírez (2007), "Desarrollo y políticas urbanas en México: retos y perspectivas”, en José Luis Calva (coord.), Políticas de desarrollo regional, Porrúa-Universidad Nacional Autónoma de México, México, pp. 353-383.

Azuela, Antonio (1989), La ciudad, la propiedad privada y el derecho, El Colegio de México, México.

вм (Banco Mundial) (2010), World development report 2010. Development and Climate Change, Banco Mundial, Washington.

CD (Cámara de Diputados) (2011), Análisis mensual de productos básicos, LXI Legislatura, Centro de Estudios de Finanzas Públicas, México.

Cepal (Comisión Económica para América Latina y el Caribe) (2008), Estudio económico de América Latina y el Caribe. Política macroeconómica y volatilidad, CEPAL, Santiago.

CEPAl (Comisión Económica para América Latina y el Caribe) (2009), La economía del cambio climático en América Latina y el Caribe, CEPAL, Santiago.

CEPAL (Comisión Económica para América Latina y el Caribe)-BID (Banco Interamericano de Desarrollo (2009), Cambio climático. Una perspectiva regional, CEPAL-BID, Santiago.

Conabio (Comisión Nacional para el Conocimiento y Uso de la Biodiversidad) (1997), "Cuencas hidrológicas" [metadatos y mapoteca digital], Conabio, México. 
Conagua (Comisión Nacional del Agua) (1998), "Cuencas hidrológicas" [metadatos y mapoteca digital], Conabio, México.

Conagua (Comisión Nacional del Agua) (2010), Estadísticas del agua en México, Conagua, México.

Conapo (Consejo Nacional de Población) (2005), Sistema urbano nacional: población y tasas de crecimiento, 1990-201, Conapo, México.

DOF (Diario Oficial de la Federación) (1997), 8 de enero.

DOF (Diario Oficial de la Federación) (2008), "Ley de Aguas Nacionales", DoF, México, 18 de abril.

Dourojeanni, Axel y Andrei Jouravlev (1999), Gestión de cuencas y ríos vinculados con centros urbanos, CEPAL, Santiago.

Dourojeanni, Axel, Andrei Jouravlev y Guillermo Chávez (2002), Gestión del agua a nivel de cuencas: teoría y práctica, CEPAL, Santiago.

FAO (Organización de las Naciones Unidas para la Agricultura y la Alimentación) (2011), El estado mundial de la agricultura y la alimentación, FAO, Roma.

Garduño, Héctor, Stephen Foster, Marcella Nanni, Karin Kemper, Albert Tuinhof y Phoebe Koundouri (2006), Groundwater Dimensions of National Water Resource and River Basin Planning. Promoting an integrated strategy, Banco Mundial, Washington.

GWP (Global Water Partnership)-INBo (International Network of Basin Organizations) (2009), A Handbook for Integrated Water Resources Management in Basins, GWP-INBO, Estocolmo.

GWsP (The Global Water System Project) (2005), The Global Water System Project: Science Framework and Implementation Activities. Earth System Science Partnership, GwsP, Estocolmo.

Hirji, Rafki y Richard Davis (2009), Strategic Environmental Assessment: Improving Water Resources Governance and Decision Making, Banco Mundial, Washington. 
IFPRI (International Food Policy Research Institute)-rWMI (International Water Management Institute) (2002), Global Water Outlook to 2025. Averting an Impending Crisis, IFPRI-IWMI, Washington.

INEGI (Instituto Nacional de Estadística, Geografía e Informática)-INE (Instituto Nacional de Ecología) (1996), Usos del suelo y vegetación [metadatos y mapoteca digital], Conabio, México.

INEGI (Instituto Nacional de Estadística, Geografía e Informática) (2005), Sistema urbano y localidades [metadatos y mapoteca digital], INEGI, México.

IBRD/BM (International Bank for Reconstruction and Development/World Bank) (2010), Sustaining water for all in a changing climate, World Bank Group Implementation Progress Report of the Water Resources Sector Strategy, Banco Mundial, Washington.

IPCC (Intergovernmental Panel on Climate Change) (2007a), Climate Change 2007. Adaptation. Contribution of Working Group III to the Fourth Assessment Report of the Intergovernmental Panel on Climate Change, IPCC, Londres.

IPCC (Intergovernmental Panel on Climate Change) (2007b), Climate Change: The Physical Science Basis. Contribution of Working Group I to the Fourth Assessment. Report of the Intergovernmental Panel on Climate Change, IPCC, Londres.

IPCC (Intergovernmental Panel on Climate Change) (2007c), Climate Change 2007. Mitigation. Contribution of Working Group III to the Fourth Assessment Report of the Intergovernmental Panel on Climate Change, IPCC, Londres.

Keller, Andrew, Ramasamy Sakthivadivel y David Seckler (2000), Water scarcity and the role of storage in development, International Water Management Institute, Colombo.

Legorreta, Jorge (2006), El agua y la ciudad de México. De Tenochtitlán a la megalópolis del siglo XXI, Universidad Autónoma Metropolitana, Azcapotzalco, México. 
Ley General de Asentamientos Humanos (1994), Diario Oficial de la Federación, 5 de agosto.

Maderey-Rascón, Laura y Cuauhtémoc Torres-Ruata (1990), Cuencas hidrológicas [metadatos y mapoteca digital], Instituto de Geografía, México.

Martínez Alier, Joan y Jordi Roca (2001), Economía ecológica y política ambiental, Fondo de Cultura Económica, México.

Mendoza, Jorge y Alejandro Díaz-Bautista (2006), Economía regional moderna. Teoría y práctica, El Colegio de la Frontera Norte, México.

Molle, Françoise (2006), Planning and management water resources at the river basin level: emergence and evolution of a concept, International Water Management Institute, Colombo.

Molle, Françoise y Chu Thai Hoanh (2009), Implementing Integrated River Basin Management: Lessons from the Red River Basin, Vietnam, International Water Management Institute, Colombo.

Monroy-Ortiz, Rafael y Rafael Monroy (2007), “Omisiones y fragmentación. El reto de la planeación”, Revista de Arquitectura, Diseño y Urbanismo, (2), Universidad Autónoma del Estado de Morelos, Cuernavaca, pp. 279-285.

ocde (Organización para la Cooperación y el Desarrollo Económicos) (2006), Competitive cities in the global economy, OCDE, París.

O'Connor, James (2001), Causas naturales. Ensayos de marxismo ecológico, Siglo XXI, México.

PNUD (Programa de las Naciones Unidas para el Desarrollo) (2009), Informe sobre el desarrollo humano 2009. Superando barreras: movilidad y desarrollos humanos, PNUD, Nueva York.

PNUd (Programa de las Naciones Unidas para el Desarrollo) (2010), The Millennium Development Goals Report 2010, pNud, Nueva York. 
Revenga, Carmen, Jake Brunner, Norbert Henninger, Ken Kassem y Richard Payne (2000), Pilot analysis of global ecosystem. Freshwater Systems, World Resources Institute, Washington.

Rijsberman, Frank y David Molden (2001), Balancing water uses: Water for food and water for nature, International Water Management Institute, Colombo.

Rosegrant, Mark W., Cai Ximing y Sarah A. Cline (2002), Global water outlook to 2025. Averting an Impending Crisis, International Water Management Institute.

Seckler, David (1996), The New Era of Water Resources Management: From "Dry" to "Wet" Water Savings, International Irrigation Management Institute, Colombo.

Sedesol (Secretaría de Desarrollo Social) (2001), Programa Nacional de Desarrollo Urbano y Ordenación del Territorio 2001-2006, Sedesol, México.

Sedesol (Secretaría de Desarrollo Social)-Conapo (Consejo Nacional de Población)-INEgi (Instituto Nacional de Estadística, Geografía e Informática) (2006), Delimitación de las zonas metropolitanas de México 2005, Sedesol, México.

Smakhtin, Vladimir, Carmen Revenga y Petra Döll (2004), Taking into Account Environmental Water Requirements in Global-scale Water Resources Assessments, International Water Management Institute, Colombo.

unep (United Nations Environment Programme) (2007), Global Environment Outlook GEO 4, UNEP, Malta.

UNESCO (Organización de las Naciones Unidas para la Educación, la Ciencia y la Cultura) (2006), The United Nations World Water Development, Report 2, Water a shared responsibility, unEsco, París.

unesco (Organización de las Naciones Unidas para la Educación, la Ciencia y la Cultura) (2009), The United Nations World Water Development. Report 3. Water in a changing world, unesco, París. 
un-Habitat (United Nations Human Settlements Programme) (2006), State of worl's cities 2006/7. The millennium developments goals and urban sustainability: 30 years of shaping the habitat agenda, un-Habitat, Nairobi.

un-Habitat (United Nations Human Settlements Programme) (2009), Planning sustainable cities: policy directions. Global report on human settlements 2009, un-Habitat, Londres.

UNPF (United Nations Population Fund) (2009), State of world population 2009. Facing a changing world: women, population and climate, UNPF, Nueva York.

un-Water (United Nations-Water) (2008), Status Report on Integrated Water Resources Management and Water Efficiency Plans, un-Water, Colombo.

wwc (World Water Council) (2009), Global water framework. Outcomes of the $5^{\text {th }}$ world water forum, wwc, Estambul.

Recibido: 2 de mayo de 2010. Reenviado: 8 de junio de 2011. Aceptado: 22 de agosto de 2011.

Rafael Monroy-Ortiz. Es doctor en economía por la Universidad $\mathrm{Na}-$ cional Autónoma de México (UNAM). Es profesor-investigador en la Facultad de Arquitectura, en el Sistema de Estudios de Posgrado e Investigación, de la Universidad Autónoma del Estado de Morelos. También pertenece al Sistema Nacional de Investigadores. Es profesor de asignatura definitivo, en el área urbano ambiental, de la Facultad de Arquitectura de la UNAM. Sus líneas de investigación son: estudios urbano-ambientales para el escenario del cambio climático. Entre sus publicaciones destacan: en coautoría, "Consideraciones sobre el aprovechamiento económico de la eterna primavera", Inventio. La Génesis de la Cultura Universitaria en Morelos, 13, UAEM, Cuernavaca, pp. 5-11 (2011); "La expansión urbana sobre el campo mexicano. La otra cara de la crisis", Estudios Agrarios, 43, Procuraduría Agraria, Distrito Federal, pp. 29-46, (2010); "La biodiversidad en el sistema urbano de cuenca. El caso de Río Grande Amacuzac", en Rafael Monroy, Hortensia Colín y Saúl Roque (eds.), Los 
pueblos de Morelos cabalgan por la vida, UAEM, Cuernavaca, pp. 69-87 (2009); "Capital inmobiliario y transformación del territorio", Ciudades, 79, Red Nacional de Investigación Urbana, Puebla, pp. 2-10 (2008); "Competitividad ambiental y competitividad urbana", Ciudades, 77, Red Nacional de Investigación Urbana, Puebla, pp. 38-46 (2008). 